\title{
Modelo de Shaw y Oxford aplicado a un Proceso de Taladrado que Utiliza Brocas Helicoidales con Geometría Innovada y Ausencia de Bisel
}

\author{
Erardo E. Leal-Muñoz ${ }^{(1,2)}$ y Mario A. Guzmán ${ }^{(1)}$ \\ (1) Depto. de Ingeniería Mecánica, Univ. de La Frontera, Francisco Salazar 1145, Temuco, Chile \\ (2) Escuela Superior de Ingenieros Industriales, Univ. Politécnica de Madrid, José Gutiérrez Abascal 2, \\ 28006, Madrid, España (e-mail: erardo.leal@ufrontera.cl; mario.guzman@ufrontera.cl)
}

Recibido Jun. 6, 2016; Aceptado Ago. 7, 2016; Versión final Sep. 7, 2016, Publicado Feb. 2017

\begin{abstract}
Resumen
En este artículo se presenta una forma corregida del modelo de Shaw y Oxford para brocas con geometría mejorada y ausencia de bisel transversal. Se ha realizado un ajuste de las expresiones que conforman el modelo y se han determinado los parámetros experimentales para estimar la fuerza de avance y el par de taladrado en aluminio Al-2011. Los resultados obtenidos mediante el modelo corregido se han comparado con valores estimados mediante el modelo de la presión específica de corte. Finalmente, se muestra que el modelo de Shaw y Oxford puede ser aplicado en brocas con geometría mejorada mediante el ajuste del parámetro asociado al bisel transversal.
\end{abstract}

Palabras clave: presión específica de corte; mecanizado; fuerza de corte; taladrado; Shaw y Oxford

\section{The Shaw and Oxford Model Applied to a Process of Drilling that uses Twist Drills with Innovated Geometry and without Chisel}

\begin{abstract}
This article presents a corrected form of the Shaw and Oxford model for drills with improved geometry and absence of the edge chisel. Adjustments of the expressions that compose the model have been made, and also the experimental parameters have been determined in order to estimate the thrust force and the torque of the drilling in aluminum Al-2011. The results obtained through the corrected model, have been compared with estimated values through the specific cutting pressure model. Finally, it is proved that the Shaw and Oxford model can be applied to drills with improved geometry through the adjustment of the parameter associated to the edge chisel.
\end{abstract}




\section{INTRODUCCIÓN}

El taladrado es una combinación de dos movimientos, uno rotativo principal y otro de avance lineal, lo que conduce a obtener un agujero de forma cilíndrica recta. Esto es logrado mayoritariamente, utilizando una broca helicoidal. Una broca helicoidal convencional (figura 1), se caracteriza fundamentalmente por tener: ángulo de la punta, ángulo entre los filos principales, ángulo del bisel transversal, ángulo de la hélice, longitud de los filos principales de corte y longitud del bisel transversal. El bisel transversal tiene una gran influencia sobre las fuerzas de corte y la calidad de los agujeros (Pirtini y Lazoglu, 2005). En una operación de taladrado el bisel transversal arranca material mediante un proceso de indentación (Isbilir y Ghassemieh, 2013), con presencia de un ángulo de desprendimiento altamente negativo.

La necesidad de estudiar mejoras en las brocas se ha vuelto importante a partir del mecanizado de materiales compuesto. Durante el proceso de taladrado en compuestos se presenta un fenómeno llamado delaminación (Hocheng y Dharan, 1990) que se produce principalmente en la salida de la broca. Para disminuir el efecto del delaminado en los materiales compuestos otros autores han implementado diversas estrategias, como refuerzos con fibra de carbono, uso de agua congelada y mejoras en la geometría de la herramienta. Li et al. (Li et al., 2015) desarrolló una técnica utilizando nano tubos de fibra de carbono, para mejorar la resistencia interlaminar y la conductividad térmica, dos factores que influyen en la delaminación. Hocheng et al. (Hocheng et al., 2016) aprovechó la capacidad de fuerza expansiva interna del agua, logrando disminuir la delaminación, lo que permite la utilización de avances más grandes, en este caso además disminuyendo tiempos de proceso. Otro de los enfoques, es el que se centra en la mejora de la geometría de la herramienta, optimizando la geometría convencional (Sambhav et al., 2012) y mejorando el perfil de los filos (Xiong et al., 2009).

Para minimizar la fuerza de avance y el par de taladrado, con enfoque en la herramienta, se ha optimizar la geometría de la punta de la herramienta, pero conservando la presencia del bisel transversal (Wang y Zhang, 2008; Wang y Zhang, 2008; Anish et al., 2005; Fetecau et al., 2009). Las mejoras realizadas en la geometría de las brocas helicoidales, corresponden a dividir al bisel transversal en dos filos de corte, creando un destalonado en cruz, estas brocas han sido afiladas de tal manera que se generan dos filos primarios los cuales generan la formación de un ángulo de desprendimiento positivo, tal como se aprecia en la figura 2. Se ha mostrado que estas brocas mejoradas generan una disminución en la fuerza de avance (Guzmán y Caire, 2014). Se reconoce que el bisel transversal de la broca aporta una significativa magnitud de la componente de la fuerza de avance (Hamade et al., 2006).

El modelado de los proceso de corte tiene por objetivo ajustar las condiciones de corte apropiadas (Durán y Bavestrello, 2006), evaluar el desgaste de la herramienta (Rehorn et al., 2005) y el consumo energético (Sarache et al., 2007) entre otros. Los métodos de modelado utilizados por diversos autores son los basados en herramientas numéricas, como los elementos finitos y mediante el desarrollo de diversos modelos mecanicistas (Yellowley, 1985, López de Lacalle et al., 2009).

En el año 1957 los investigadores Shaw y Oxford (Shaw y Oxford, 1957) establecieron que el proceso de taladrado puede ser dividido en dos partes. Una es la acción de los filos principales de corte y la otra es la acción del bisel transversal. Los modelos propuestos por Shaw y Oxford fueron desarrollados para el mecanizado de metales, además han demostrado su aplicabilidad en materiales compuestos (Okutan et al, 2013; Fernandes y Cook, 2007). Karabay (karavay 2007) utilizó el modelo como base para desarrollar un sistema de medición para taladrado. Para predecir la fuerza de avance $F_{f}$ y el par de corte $M_{c}$, Shaw y Oxford formularon el proceso a través de la ecuación (1) y ecuación (2) respectivamente.

$$
\begin{aligned}
& \frac{F_{f}}{D^{2} H_{B}}=k_{1} \frac{s^{2 a} f_{n}^{1-a}}{D^{1+a}}\left(\frac{1-\frac{c}{D}}{\left(1+\frac{c}{D}\right)^{2}}+k_{2}\left(\frac{c}{D}\right)^{1-a}\right)+k_{3}\left(\frac{c}{D}\right)^{2} \\
& \frac{M_{c}}{D^{3} H_{B}}=k_{4} \frac{s^{2 a} f_{n}^{1-a}}{D^{1+a}}\left(\frac{1-\left(\frac{c}{D}\right)^{2}}{\left(1+\frac{c}{D}\right)^{a}}+k_{5}\left(\frac{c}{D}\right)^{2-a}\right)
\end{aligned}
$$

El cálculo de la fuerza de avance $F_{f}$ y del par de corte $M_{c}$ están en función de la dureza del material mecanizado $H_{B}$, del diámetro de la broca $D$, del avance de la herramienta $f_{n}$, de la longitud del bisel transversal $\mathrm{c}$ y del factor $\mathrm{s}$, cuyo valor promedio representa la imperfección del material que se pretende mecanizar. Los valores de: $\mathrm{s}, \mathrm{a}, \mathrm{k}_{1}, \mathrm{k}_{2}, \mathrm{k}_{3}, \mathrm{k}_{4}$ y $\mathrm{k}_{5}$, son constantes que deben ser determinadas a través de actividades experimentales. 

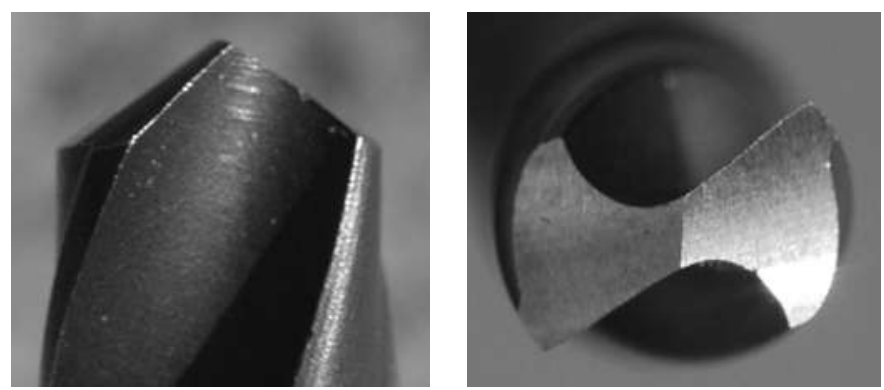

Fig. 1: Broca con geometría convencional.
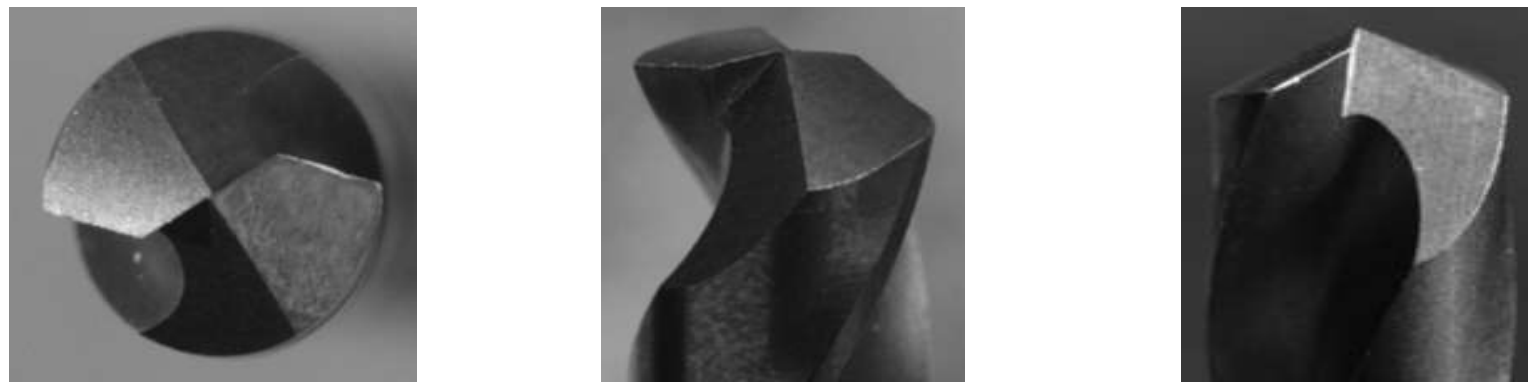

Fig. 2: Vistas de una broca con geometría mejorada en la punta.

En la ecuación (1) y ecuación (2), el factor (c/D), representa la influencia del bisel transversal en el cálculo de la fuerza de avance $F_{f}$ y del par de taladrado $M_{c}$. Por otra parte, los modelos basados en la presión específica de corte orientados a predecir la fuerza de avance $F_{f}$ y la fuerza de corte $F_{c}$, han sido ampliamente utilizados tanto para proceso de taladrado como para fresado (Yellowley, 1985; Lazar y Xirouchakis, 2013; Rey et al., 2016). La formulación del método está secuencialmente expresado en las ecuaciones (3) y (4).

$\mathrm{F}_{\mathrm{f}}=\mathrm{k}_{\mathrm{f}} \mathrm{A}$

$\mathrm{F}_{\mathrm{c}}=\mathrm{k}_{\mathrm{c}} \mathrm{A}$

Donde $k_{f}$ es el valor de la presión específica de corte asociada a la fuerza de avance, $k_{c}$ es el valor de la presión específica asociada a la fuerza de corte y A corresponde al área de corte no deformada. Como consecuencia de conocer la fuerza de corte $F_{c}$ que actúa en cada filo de la broca y la distancia que las separa, se logra calcular el momento de taladrado $M_{c}$.

En este trabajo se busca probar que las ecuaciones originales de Shaw y Oxford destinadas a modelar la fuerza de avance y el par de taladrado, tienen validez cuando se utilizan en brocas sin bisel transversal, donde el factor (C/D) tiende a cero.

\section{METODOLOGÍA}

Para realizar los ensayos de taladrado se dispuso de los siguientes recursos: i) Máquina herramienta CNC marca Hercus, modelo VMC300 (figura 3); ii) Sensores piezoeléctricos para fuerza y torque Kistler 9336A/D, 9211B con amplificador Kistler 5073; iii) Sistema de adquisición de datos National Instruments PXI-8108 con bloque de conectores NI CB-68LP; iv) Brocas con geometría innovada en las puntas. Herramientas de diámetro 4, 5, 6, 7 y 8 mm; y v) Probetas de aleación de aluminio Al-2011 (Dureza 95 HB).

\section{Condiciones de corte en taladrado}

Considerando las propiedades del material a taladrar y el diámetro de las brocas, se determinó la velocidad rotacional $n$, el avance axial $f_{n}$, la velocidad de avance $V_{f}$ y la velocidad de corte $V_{c}$ en base a la ecuaciones (5), (6), (7) y (8), respectivamente. 


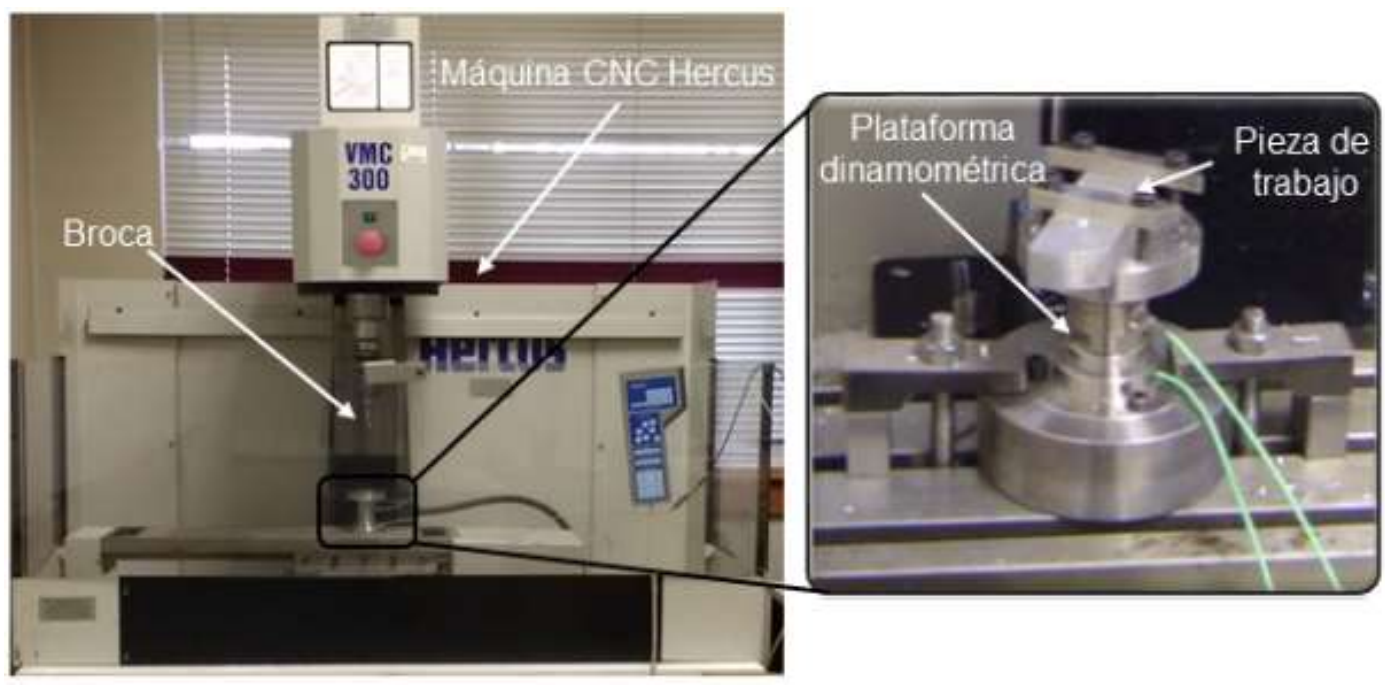

Fig. 3: Esquema de los ensayos de taladrado.

$\mathrm{n}=11254 \mathrm{D}^{-0,8353}$

$f_{n}=0,0159 D^{1,0789}$

$\mathrm{V}_{\mathrm{f}}=\mathrm{f}_{\mathrm{n}} \mathrm{n}$

$V_{c}=\frac{\pi D n}{1000}$

La tabla 1 registra las condiciones cinemáticas determinadas para realizar los ensayos de taladrado considerando los distintos diámetros seleccionados.

Tabla 1: Condiciones de corte para los ensayos.

\begin{tabular}{|c|c|c|c|c|}
\hline Diámetro D (mm) & $\begin{array}{c}\text { Velocidad rotacional } \\
\mathrm{N}(\mathrm{rev} / \mathrm{min})\end{array}$ & $\begin{array}{c}\text { Avance axial } \\
\mathrm{f}_{\mathrm{n}}(\mathrm{mm} / \mathrm{rev})\end{array}$ & Velocidad de Avance axial $\mathrm{V}_{\mathrm{f}}(\mathrm{mm} / \mathrm{min})$ & $\begin{array}{c}\text { Velocidad de Corte } \mathrm{V}_{\mathrm{c}} \\
(\mathrm{m} / \mathrm{min})\end{array}$ \\
\hline 4 & 3535 & 0,07 & 251 & 44 \\
\hline 5 & 2934 & 0,09 & 265 & 46 \\
\hline 6 & 2520 & 0,11 & 277 & 48 \\
\hline 7 & 2215 & 0,13 & 287 & 49 \\
\hline 8 & 1981 & 0,15 & 297 & 50 \\
\hline
\end{tabular}

Tipo de brocas utilizadas

En la tabla 2 se muestran las características geométricas de las brocas ensayadas, cuyo material corresponde a un acero rápido (HSS). En la figura 4 está representada la geometría más importante de las brocas innovadas. Dichas mejoras están centradas fundamentalmente, en la parte activa de la herramienta.

Tabla 2: Características geométricas de las brocas ensayadas.

Angulo de desprendimiento del filo transversal $\gamma_{\mathrm{i}}: 9^{\circ}$
Angulo de la hélice $\gamma_{\mathrm{f}}: 26^{\circ}$
Angulo entre filos principales $\sigma: 140^{\circ}$
Angulo del filo transversal $\psi: 45^{\circ}$
Altura del filo $: Z$

\section{Método de la presión específica de corte}

A modo de ejemplo ilustrativo, se muestran los resultados obtenidos al taladrar con una broca de $6 \mathrm{~mm}$ de diámetro. Los resultados que se presentan a continuación, fueron graficados con datos medidos y calculados, los que corresponden a la presión específica de la fuerza de avance en el filo transversal (figura 
5), presión específica de la fuerza de avance en el filo principal (figura 6), fuerza de avance axial (figura 7), presión específica de corte (figura 8), fuerza de corte (figura 9) y par de taladrado (figura 10). Todos los gráficos están referidos a lo que ocurre en la parte activa de la broca.

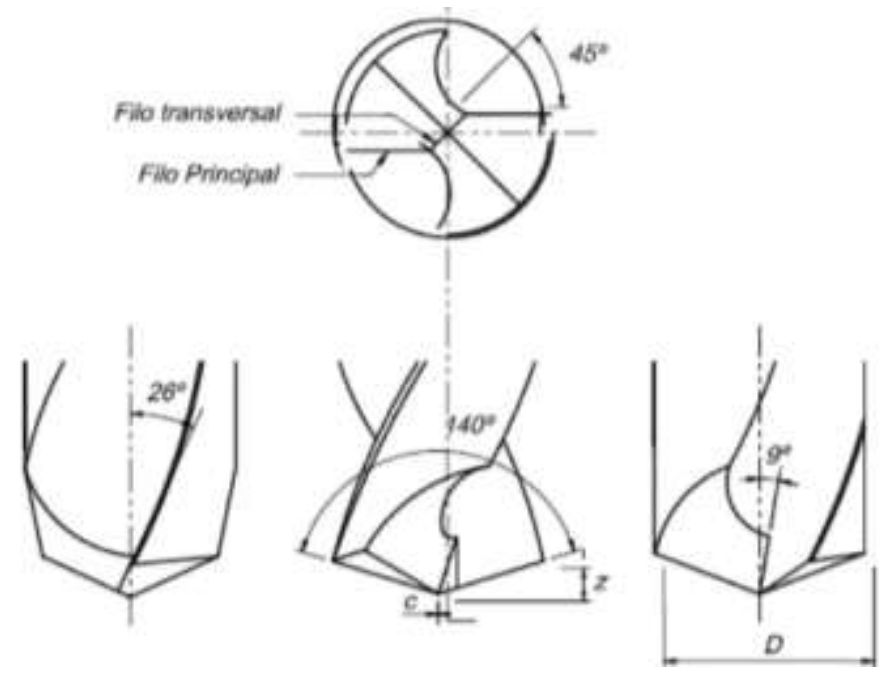

Fig. 4: Geometría de las brocas innovadas, principalmente en su parte activa.

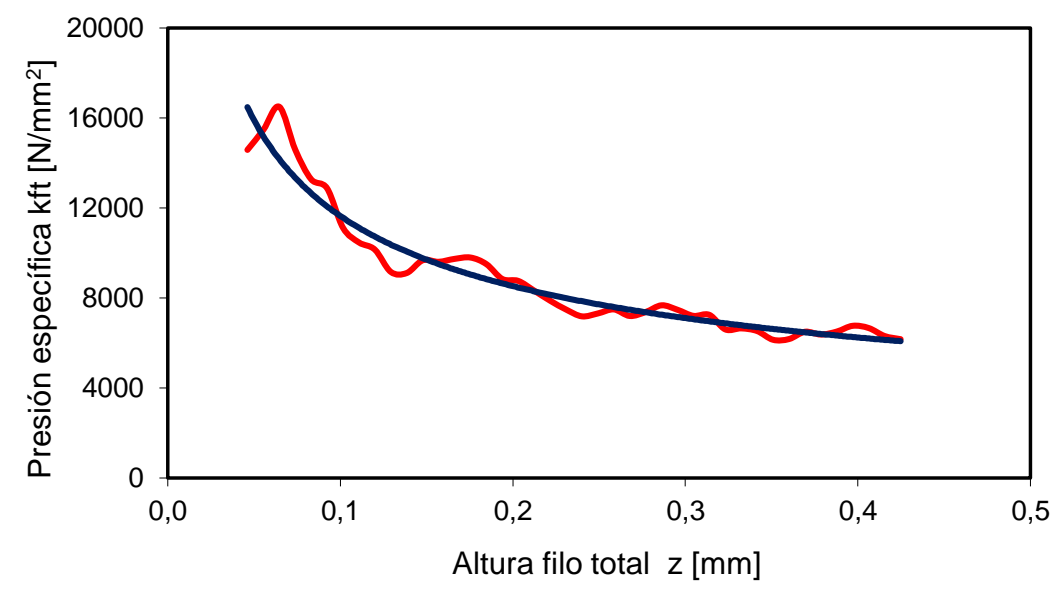

Fig. 5: Presión específica de la fuerza de avance en el filo transversal, en función de la altura del filo transversal.

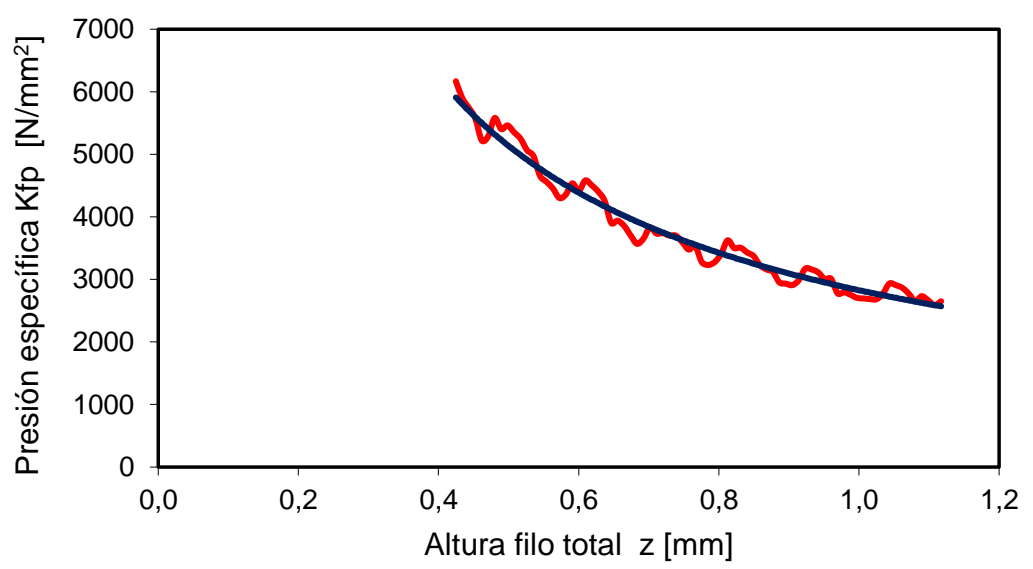

Fig. 6: Presión específica de la fuerza de avance en el filo principal, en función de la altura del filo principal. 


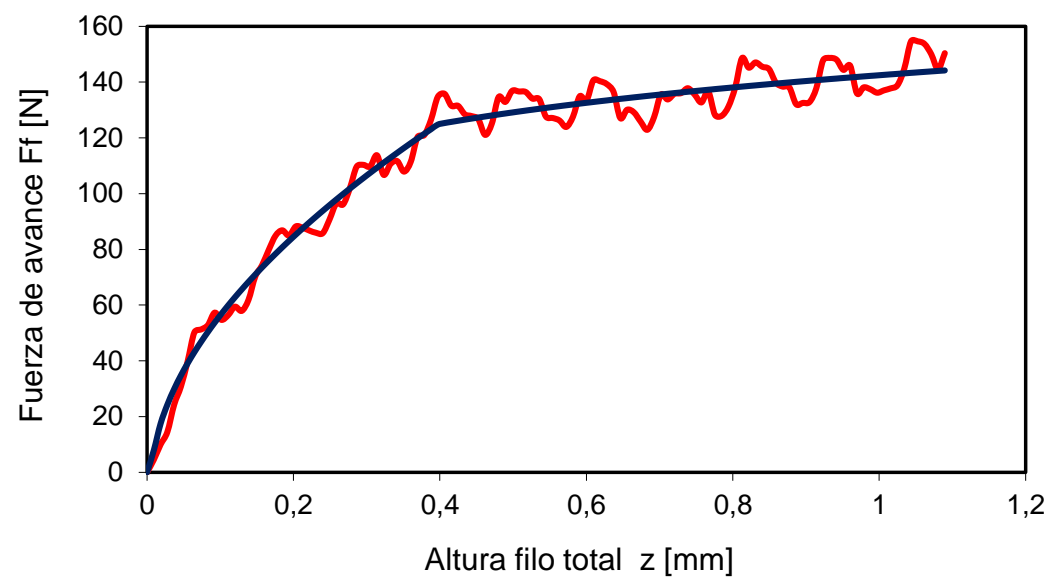

Fig. 7: Fuerza de avance axial total en función de la altura del filo.

La figura 8 representa la fuerza de avance axial, la que está compuesta por la fuerza que actúa en el filo transversal más la fuerza que actúa en el filo principal. El valor máximo de la fuerza de avance axial y el valor máximo del par de taladrado, están correlacionados con la altura máxima de la parte activa de la herramienta.

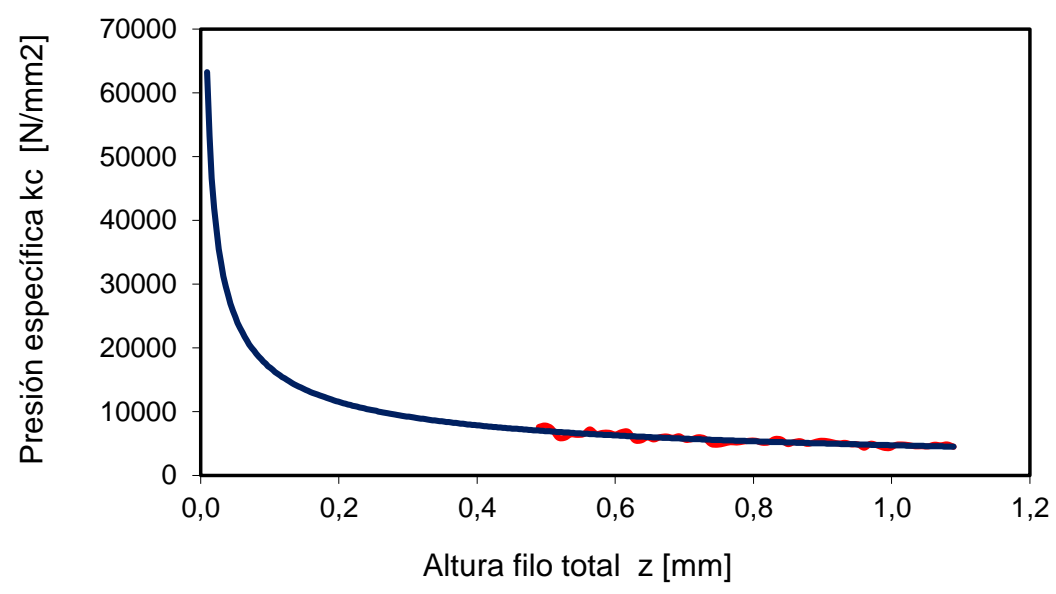

Fig. 8: Presión específica de corte en función de la altura del filo.

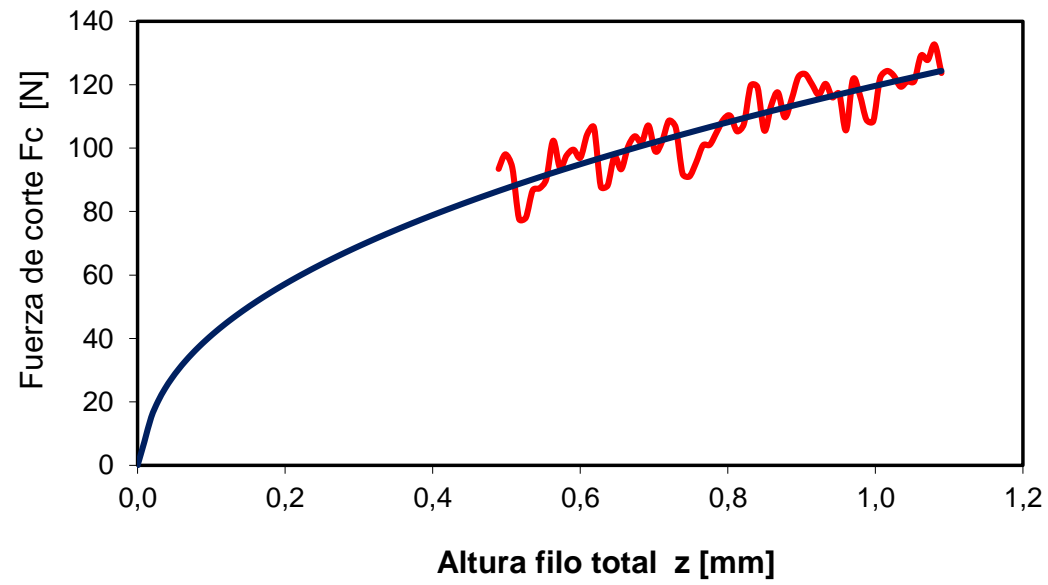

Fig. 9: Fuerza de corte en función de la altura del filo. 


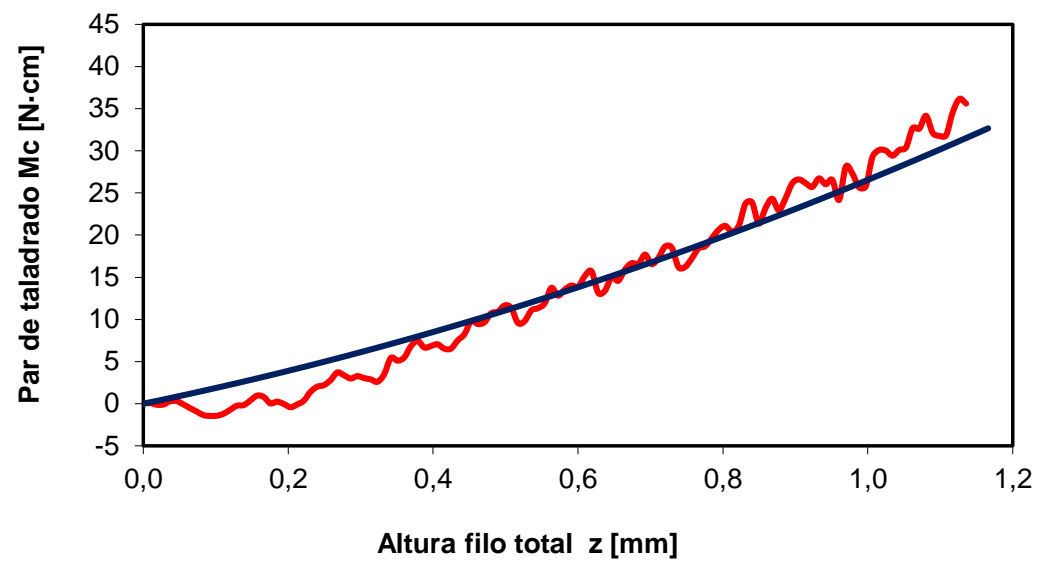

Fig. 10: Par de taladrado en función de la altura del filo.

La tabla 3 recoge los valores máximos de las fuerzas de avance axial y de los pares de taladrado para los distintos diámetros de brocas ensayadas.

Tabla 3. Resultados de los valores máximos de las fuerzas de avance y pares de taladrado.

\begin{tabular}{|c|c|c|c|}
\hline Diámetro D $(\mathrm{mm})$ & Altura del filo Z $(\mathrm{mm})$ & Fuerza de avance axial $\mathrm{F}_{\mathrm{f}}(\mathrm{N})$ & Par de taladrado $\mathrm{M}_{\mathrm{c}}(\mathrm{N} \cdot \mathrm{cm})$ \\
\hline 4 & 0,728 & 90,180 & 12,860 \\
\hline 5 & 0,910 & 112,756 & 25,817 \\
\hline 6 & 1,092 & 151,114 & 38,985 \\
\hline 7 & 1,274 & 190,203 & 55,679 \\
\hline 8 & 1,456 & 211,763 & 74,403 \\
\hline
\end{tabular}

\section{Método de Shaw y Oxford}

En la tabla 4 se encuentran los datos básicos y derivados que participan en el modelo de Shaw y Oxford. Cabe destacar la presencia de la energía específica efectiva que se genera en un proceso de taladrado, la que se expresa por la función $u=8 \mathrm{M}_{\mathrm{c}} / \mathrm{f}_{\mathrm{n}} \mathrm{D}^{2}$.

Tabla 4: Datos básicos y derivados que participan en el modelo de Shaw y Oxford.

\begin{tabular}{|c|c|c|c|c|c|c|}
\hline $\mathrm{D}(\mathrm{mm})$ & $f_{\mathrm{n}}(\mathrm{mm} / \mathrm{rev})$ & $\mathrm{M}_{\mathrm{c}}(\mathrm{N} \cdot \mathrm{cm})$ & $\begin{array}{c}8 \mathrm{M}_{\mathrm{c}} / \mathrm{f}_{\mathrm{n}} \cdot \mathrm{D}^{2} \\
\left(\mathrm{~N} \cdot \mathrm{mm} / \mathrm{mm}^{3}\right)\end{array}$ & $\begin{array}{c}\mathrm{D} \cdot \mathrm{f}_{\mathrm{n}} \\
\left(\mathrm{mm}^{2} / \mathrm{rev}\right)\end{array}$ & $\begin{array}{c}\mathrm{Ln}\left(8 \mathrm{M}_{\mathrm{c}} / \mathrm{f}_{\mathrm{n}} \cdot \mathrm{D}^{2}\right) \\
\left(\mathrm{N} \cdot \mathrm{mm}^{2} / \mathrm{mm}^{3}\right)\end{array}$ & $\begin{array}{c}\mathrm{In}^{3}\left(\mathrm{D} \cdot \mathrm{f}_{\mathrm{n}}\right) \\
\left(\mathrm{mm}^{2} / \mathrm{rev}\right)\end{array}$ \\
\hline 4 & 0,07 & 12,860 & 918,571 & 0,28 & 6,823 & $-1,273$ \\
\hline 5 & 0,09 & 25,817 & 917,950 & 0,45 & 6,822 & $-0,799$ \\
\hline 6 & 0,11 & 38,985 & 787,576 & 0,66 & 6,669 & $-0,416$ \\
\hline 7 & 0,13 & 55,679 & 699,267 & 0,91 & 6,550 & $-0,094$ \\
\hline 8 & 0,15 & 74,403 & 620,025 & 1,20 & 6,430 & 0,182 \\
\hline
\end{tabular}

Si la razón (c/D) se hace igual a cero y el factor $s=1$, las ecuaciones (1) y (2) pasan a convertirse en las ecuaciones (9) y (10), donde los coeficientes quedan reducidos a $K_{1}, K_{2}$ y $K_{3}$, respectivamente.

$F_{f}=K_{1} H_{B}\left(D f_{n}\right)^{1-a}+K_{2} H_{B} D^{2}$

$M_{C}=K_{3} H_{B} f_{n}^{1-a} D^{2-a}$

La figura 11 se construye en base a los datos de las dos últimas columnas de la tabla 4. La regresión lineal ajustada al gráfico muestra una pendiente cuyo valor es $(a=0,2838)$. Este valor permite determinar los exponentes de las ecuaciones (9) y (10). Es decir: $(1-a)=0,7162 ;(1+a)=1,2838$ y $(2-a)=1,7162$. La disposición de los datos en la forma que se presentan en la tabla 5, conlleva a trazar la figura 12 y la figura 13. La figura 12 y figura 13 responden a un ajuste de correlación lineal, de donde es posible extraer coeficientes $\mathrm{K}_{1}, \mathrm{~K}_{2}$ y K $\mathrm{K}_{3}$, respectivamente. 


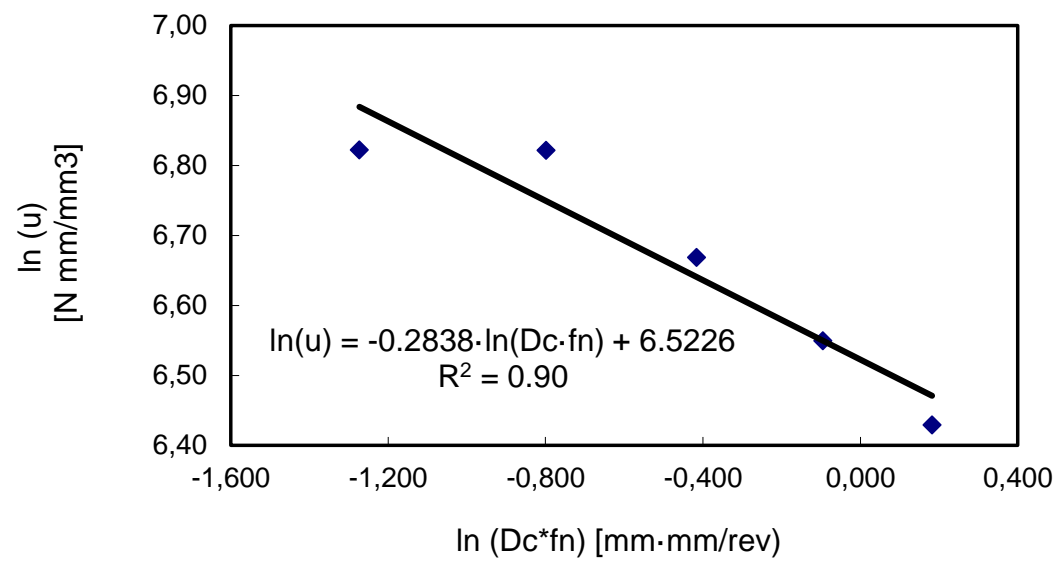

Fig. 11: Comportamiento de la energía específica $(u)$ con respecto al factor $\left(D \cdot f_{n}\right)$.

Tabla 5: Datos para producir las figuras 12 y 13.

\begin{tabular}{|c|c|c|}
\hline$\frac{F_{f}}{D^{2} H_{B}}$ & $\frac{M_{c}}{D^{3} H_{B}}$ & $\frac{F_{n}^{(0,7162)}}{D^{(1,2838)}}$ \\
\hline 0,05933 & 0,00212 & 0,02512 \\
\hline 0,04748 & 0,00217 & 0,02258 \\
\hline 0,04419 & 0,00190 & 0,02063 \\
\hline 0,04086 & 0,00171 & 0,01908 \\
\hline 0,03483 & 0,00153 & 0,01780 \\
\hline
\end{tabular}

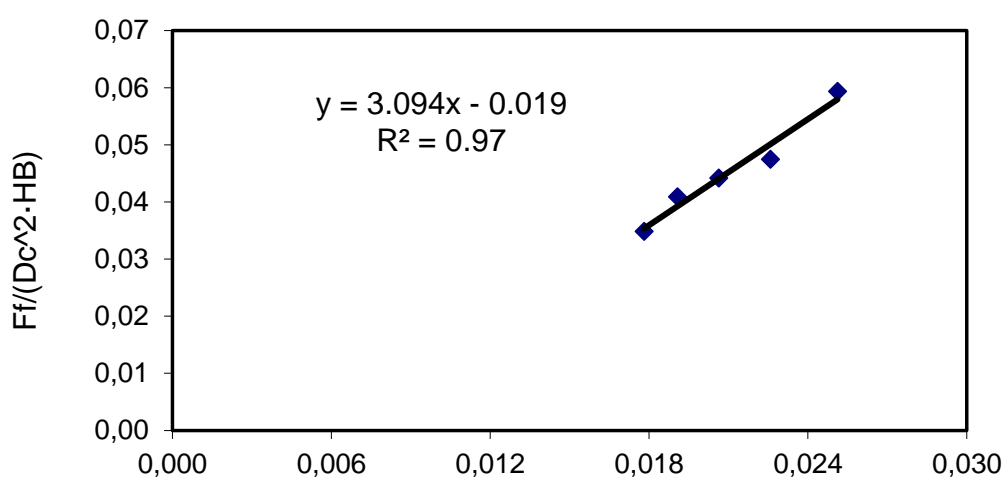

$f^{\wedge} 0,7162 / D c^{\wedge} 1,2838$

Fig. 12: Obtención de los coeficientes $\mathrm{K}_{1}$ y $\mathrm{K}_{2}$.

Los coeficientes $K_{1}, K_{2}$ y $K_{3}$, juntos a la dureza del material $H_{B}$, el avance de corte $F_{n}$ y el diámetro de la broca D, participan en las ecuaciones (9) y (10), que permiten calcular las fuerzas de avance $F_{f}$ y los pares de taladrado $\mathrm{M}_{\mathrm{c}}$. La Tabla 6 recoge los valores obtenidos mediante el método de Shaw y Oxford y el método de la presión específica de corte.

Tabla 6: Valores de las fuerzas de avance y pares de taladrado obtenidos mediante los métodos de Shaw y Oxford y de la Presión Específica de Corte.

\begin{tabular}{|c|c|c|c||c|c|c|}
\hline $\mathrm{D}(\mathrm{mm})$ & Shaw/Oxford Ff (N) & Presión Específica Ff (N) & $\Delta \mathrm{Ff}(\mathrm{N})$ & $\begin{array}{c}\text { Shaw/Oxford } \\
\text { Mc }(\mathrm{N}-\mathrm{cm})\end{array}$ & $\begin{array}{c}\text { Presión Específica Mc } \\
(\mathrm{N}-\mathrm{cm})\end{array}$ & $\begin{array}{c}\Delta \mathrm{Mc}(\mathrm{N}- \\
\mathrm{cm})\end{array}$ \\
\hline 4 & 86,953 & 90,180 & 3,23 & 12,979 & 12,860 & 0,12 \\
\hline 5 & 117,223 & 112,756 & 4,47 & 22,853 & 25,817 & 3,03 \\
\hline 6 & 148,164 & 151,114 & 2,95 & 36,132 & 38,985 & 3,01 \\
\hline 7 & 179,305 & 190,203 & 10,90 & 53,124 & 55,679 & 2,85 \\
\hline 8 & 210,290 & 211,763 & 1,47 & 74,097 & 74,403 & 0,79 \\
\hline
\end{tabular}




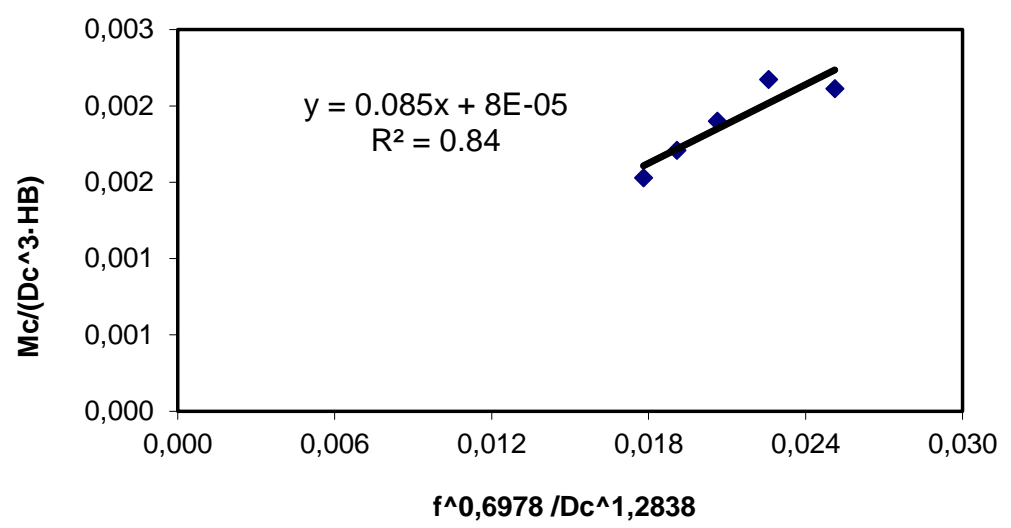

Fig. 13: Obtención del coeficiente $K_{3}$.

\section{RESULTADOS}

La figura 14 representa los resultados de las fuerzas de avance en taladrado para los métodos de Shaw y Oxford y de la presión específica de corte. La figura 15 representa los resultados de los pares de taladrado para los métodos de Shaw y Oxford y de la presión específica de corte.

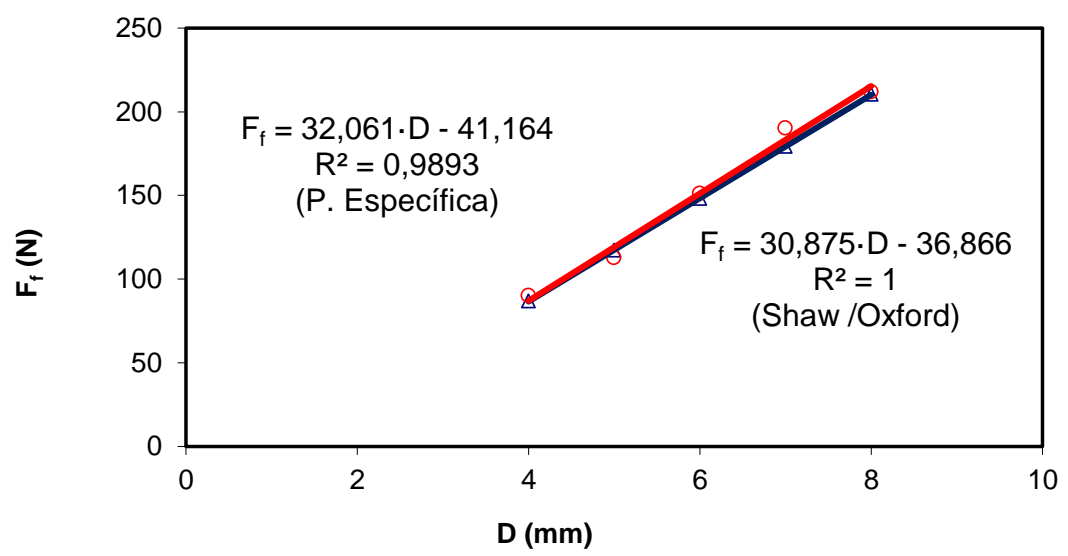

Fig. 14: Fuerzas de avance calculadas por los métodos de Shaw y Oxford y de la presión específica de corte. $\Delta$ Shaw y Oxford, o presión específica de corte.

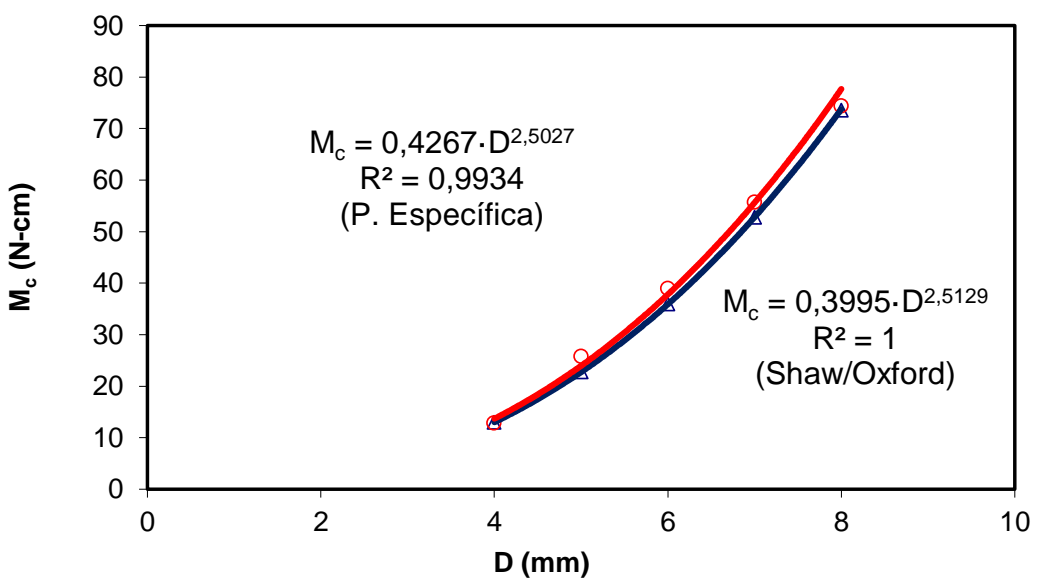

Fig. 15: Pares de taladrado calculados por los métodos de Shaw y Oxford y de la presión específica de corte. $\Delta$ Shaw y Oxford, o presión específica de corte. 


\section{CONCLUSIONES}

A partir del trabajo realizado y de los resultados expuestos, es posible concluir lo siguiente:

Para obtener los valores de referencia de las fuerzas de avance y de los pares de taladrado, se recurrió a valores medidos y calculados por el método de la presión específica de corte.

Anulando el factor que representa la influencia del bisel transversal, se reescribieron las ecuaciones originales de Shaw y Oxford, logrando ecuaciones abreviadas para las fuerzas de avance y pares de taladrado.

Al utilizar las nuevas ecuaciones de Shaw y Oxford para calcular las fuerzas de avance y par de taladrado, los resultados obtenidos muestran una cercanía cuantitativa entre los dos métodos comparados.

Se evidencia, a partir de los resultados, que es posible aplicar del modelo de Shaw y Oxford a brocas con geometría mejorada en que hay ausencia del bisel transversal y presencia del filo transversal.

\section{AGRADECIMIENTOS}

Los autores agradecen a la Dirección de Investigación de la Universidad de La Frontera, que mediante el proyecto DIUFRO DI10-0012 financió este trabajo de investigación.

\section{REFERENCIAS}

Anish, P., G. Shiv y otros dos autores, Chisel edge and cutting lip shape optimization for improved twist drill point design, doi: 10.1016/j.ijmachtools.2004.09.010, Int. J. Mach. Tools Manuf.,(en línea), 45, 421-431 (2005)

Durán, O. M. y I. Bavestrello, Definición de condiciones para taladrado usando un enfoque basado en lógica difusa, doi: 10.4067/S0718-07642006000500004, Inf. Tecnol., (en línea), 17, 15-20 (2006)

Fernandes M. y C. Cook, Drilling of carbon composites using a one shot drill bit. Part II: empirical modeling of maximum thrust force, doi: 10.1016/j.jimachtools.2005.03.016, Int. J. Mach. Tools Manuf., (en línea), 46, 76-79 (2007)

Fetecau C., F. Stan y N. Oancea, Toroidal grinding method for curved cutting edge twist drills, doi: 10.1016/j.jmatprotec.2008.08.003, J. Mater. Process. Tech., (en línea), 209, 3460-3468 (2009)

Guzmán, M.A. y F. Caire, Estudio de las fuerzas de avance en un proceso de taladrado utilizando brocas con geometría mejorada, doi: 10.4067/S0718-07642014000100003, Inf. Tecnol., (en línea), 25, 15-22 (2014)

Hamade R.F., C.Y. Seif y F. Ismail, Extracting cutting force coefficients from drilling experiments, doi: 10.1016/j.ijmachtools.2005.05.016, Int. J. Mach. Tools Manuf., (en línea), 46, 387-396 (2006)

Hocheng H. y C.K.H. Dharan, Delamination during drilling in composite laminates, doi: 10.1115/1.2899580, ASME J. Eng. Ind., (en línea), 112, 236-239 (1990)

Hocheng H. y C.C. Tsao, H.T. Chen, Utilizing internal icing force to reduce delamination in drilling composite tubes, doi: 10.1016/j.compstruct.2015.11.043, Compos. Struct., (en línea), 139, 36-41 (2016)

Isbilir O. y E. Ghassemieh, Numerical Investigation of Effect of Drill Geometry on Drilling Induced Delamination of Carbon Fiber Reinforced Composites, doi: 10.1016/j.compstruct.2013.04.026, Compos. Struct., (en línea), 105, 126-133 (2013)

Karabay S, Analysis of drill dynamometer with octagonal ring type transducers for monitoring of cutting forces in drilling and allied process, doi: 10.1016/j.matdes.2005.07.008, Mater. Design., (en línea), 28, 673$685(2007)$

Lazar M-B. y P. Xirouchakis, Mechanical load distribution along the main cutting edges in drilling, doi: 10.1016/j.jmatprotec.2012.09.020, J. Mater. Process. Tech., (en línea), 213, 245-260 (2013)

Li N., Y. Li y otros tres autores, Drilling delamination and thermal damage of carbon nanotube/carbon fiber reinforced epoxy composites processed by microwave curing, doi: 10.1016/j.jimachtools.2015.06.005, Int. J. Mach. Tools Manuf., (en línea), 97, 11-17 (2015) 
López de Lacalle L.N., A. Rivero y A. Lamikiz, Mechanistic model for drills with double point-angle edges, doi: 10.1007/s00170-007-1362-8, A. Int. Adv. Manuf. Technol., (en línea), 40, 447-457 (2009)

Okutan E., S. Karabay y otros dos autores, A Study on the Derivation of Parametric Cutting Force Equations in Drilling of GFRP Composites, doi: 10.5545/sv-jme.2012.774, J. Mech. Eng., (en línea), 59, 97-105 (2013)

Pirtini M. y I. Lazoglu. Forces and quality in drilling, doi: 10.1016/j.jmachtools.2005.01.004, Int. J. Mach. Tools Manuf., (en línea), 45, 1271-1281 (2005)

Rehorn A.G., J. Jiang y P.E. Orban, State-of-the-art methods and results in tool condition monitoring: A review, doi: 10.1007/s00170-004-2038-2, Int. J. Adv. Manuf. Technol., (en línea), 26, 693-710 (2005)

Rey P.R., J. LeDref y otros dos autores, Modelling of Cutting forces in orbital drilling of titanium alloy Ti-6Al4V, doi: 10.1016/j.ijmachtools.2016.04.006, Int. J. Mach. Tools Manuf., (en línea), 106, 75-88 (2016)

Sambhav K., P. Tandon y S.G. Dhande, Geometric Modeling and Validation of Twist with a Generic Point Profile, doi: 10.1016/j.apm.2011.08.034, Appl. Math. Model., (en línea), 36, 2384-2403 (2012).

Sarache L.M., R. Ramírez y V.M. Guédez, Análisis Multifactorial Del Consumo Energético de un Proceso de Fresado Frontal, doi: 10.4067/S0718-07642007000100010, Inf. Tecnol., (en línea), 18, 67-72 (2007)

Shaw M.C. y C.J. Oxford, On the Drilling of Metals 2- The torque and thrust in drilling, T. of the ASME, 79, 139-148 (1957)

Wang J. y Q. Zhang, A study of high-performance plane rake faced twist drills. Part I: Geometrical analysis and experimental investigation, doi: 10.1016/j.jmachtools.2008.03.005, Int. J. Mach. Tools Manuf., (en línea), 48, 1276-1285 (2008)

Wang J. y Q. Zhang, A study of high-performance plane rake faced twist drills. Part II: Predictive force models, doi: 10.1016/j.jjmachtools.2008.03.006, Int. J. Mach. Tools Manuf., (en línea), 48, 1286-1295 (2008)

Xiong L., N. Fang y H. Shi, A New Methodology for Designing a Curve-Edge Twist Drill whit an Arbitrary Given Distribution of the Cutting Angles Along the Tool Cutting Edge, doi: 10.1016/j.jimachtools.2009.01.006, Int. J. Mach. Tools Manuf., (en línea), 49, 667-677 (2009).

Yellowley I., Observations on the mean values of forces, torque and specific power in the peripherical milling process, doi: 10.1016/0020-7357(85)90034-4, Int. J. Mach. Tools Res., (en línea), 24, 337-346 (1985) 
